EPJ manuscript No.

(will be inserted by the editor)

Rapid Note

\title{
A thin film model for corotational Jeffreys fluids under strong slip
}

\author{
Andreas Münch ${ }^{1}$, Barbara Wagner ${ }^{2}$, Markus Rauscher ${ }^{3,4}$, and Ralf Blossey ${ }^{5}$ \\ 1 Institute of Mathematics, Humboldt University of Berlin, 10099 Berlin, Germany \\ 2 Weierstrass Institute for Applied Analysis and Stochastics (WIAS), Mohrenstr. 39, 10117 Berlin, Germany \\ 3 Max-Planck-Institut für Metallforschung, Heisenbergstr. 3, 70569 Stuttgart, Germany \\ 4 Institut für Theoretische und Angewandte Physik, Universität Stuttgart, Pfaffenwaldring 57, 70569 Stuttgart \\ 5 Biological Nanosystems Group, Interdisciplinary Research Institute, c/o IEMN Avenue Poincaré BP 60069, F-59652 Villeneuve \\ d'Ascq, France
}

Version: October 18, 2018

Abstract. We derive a thin film model for viscoelastic liquids under strong slip which obey the stress tensor dynamics of corotational Jeffreys fluids.

PACS. 83.60.Bc Viscoelasticity $-47.50 .+\mathrm{d}$ Non-newtonian fluid flows $-68.15 .+\mathrm{e}$ Liquid thin films

Dewetting liquid polymer films on nonwetting substrates, such as silicone wafers grafted with a monolayer of brushes, play a prominent role in many nanotechnological applications. It is known that for these situations, polymer films on the scale of a few hundred nanometers typically show large slippage [1]. Furthermore, for highly entangled polymers, the assumption of a Newtonian fluid will seize to be valid. To understand the interplay of viscous and viscoelastic properties of liquid polymers on hydrophobically coated substrates, there is a need for refined theoretical methods that are able to capture and evolve the emerging morphologies and their longtime dynamics. Dimension-reduced thin film models have shown in the past to be extremly successful to enable quantitative predictions that are hardly being attained simply via the underlying free-boundary problem.

In this paper we make an important step in that direction by developing a new thin film model that combines large slippage with viscoelastic properties. In [2] a family of thin film models ranging from weak to strong slip regimes could be derived depending on the order of magnitude of the slip length (see also [3]). Modelling the viscoelastic properties of such polymers generalized Maxwell and Jeffreys models have been widely used. In 44 a weak slip model could be combined with the linearized Jeffreys model to discuss effects of viscoelastic relaxation. More recently [5] we have shown that the strong slip limit can also be recovered for the linear Jeffreys model. In this Rapid Note we show that, for the strong slip regime, we are able to fully incorporate the general corotational Jeffreys model into our thin film model.

We begin by presenting the underlying free boundary problem for incompressible, viscous flow with velocity $\mathbf{u}$,

$$
\begin{gathered}
\boldsymbol{\nabla} \cdot \mathbf{u}=0 \\
\rho \frac{\mathrm{d} \mathbf{u}}{\mathrm{d} t}=-\boldsymbol{\nabla} p+\boldsymbol{\nabla} \cdot \boldsymbol{\tau},
\end{gathered}
$$

where we assume that the traceless part of the symmetric stress tensor $\boldsymbol{\tau}$ obeys the corotational Jeffreys model [

$$
\tau+\lambda_{1} \frac{D \tau}{D t}=\mu\left(\dot{\gamma}+\lambda_{2} \frac{D \dot{\gamma}}{D t}\right)
$$

Here, $D / D t$ denotes the Jaumann derivative which for arbitrary tensor fields $\Lambda$ is given by

$$
\frac{D \Lambda}{D t}=\frac{d \Lambda}{d t}+\frac{1}{2}(\boldsymbol{\omega} \Lambda-\Lambda \boldsymbol{\omega})
$$

where $\dot{\gamma}$ and $\boldsymbol{\omega}$ denote the rate of strain tensor and the vorticity tensor, given by

$$
\dot{\gamma}=\nabla \mathbf{u}+(\nabla \mathbf{u})^{\dagger}, \quad \boldsymbol{\omega}=\nabla \mathbf{u}-(\nabla \mathbf{u})^{\dagger},
$$

respectively; $d / d t$ is the material derivative $\partial_{t}+\mathbf{u} \cdot \nabla$. We assume the viscosity $\mu$ as well as the relaxation parameters $\lambda_{1}, \lambda_{2}$ to be constant material parameters. 
As boundary conditions we assume that the substrate is impermeable $\mathbf{u} \cdot \hat{\boldsymbol{e}}_{\mathbf{z}}=\mathbf{0}$ at $z=0$, and further the Navierslip boundary condition

$$
\mathbf{u} \cdot \hat{e}_{\|}=\frac{\mathbf{b}}{\mu} \tau \cdot \hat{e}_{\|}
$$

with a unit-vector $\hat{\boldsymbol{e}}_{\|}$parallel to the substrate (i.e., in $x$ or $y$-direction). At the liquid surface the normal component of the stress is balanced by the Laplace pressure (with surface tension coefficient $\gamma$ ) and the disjoining pressure $V(z)$, while the tangential components of the stress tensor are zero. In the following we use the reduced pressure $p_{R}=$ $p+V(z)$.

To simplify the algebra we restrict the calculations to follow to the $2 \mathrm{D}$ case, where we denote the velocity field components $\mathbf{u}=(u, w)$. We employ the strong-slip scaling, as in 2. In this limit, the friction between the liquid and the substrate is too weak to maintain a non-zero $x z$-shear stress to lowest order, and lateral pressure gradients are balanced by the $x x$-component of the stress tensor. For the stress tensor we use the same scalings as in 4], which means we assume that corresponding components of the strain rate and stress tensor are of the same order. Hence, the dimensional stress tensor reads in terms of the nondimensional stress tensor components

$$
\frac{\mu}{T}\left(\begin{array}{cc}
\tau^{x x} & \frac{\tau^{x z}}{\varepsilon} \\
\frac{\tau^{x z}}{\varepsilon} & \tau^{z z}
\end{array}\right)
$$

where $\mu$ denotes viscosity and $T$ denotes the time scale, given by $T=U / L$. $U$ denotes the characteristic velocity scale, such as the dewetting speed and $L$ the relative scale of the lateral extension of the dewetting rim. If $H$ denotes the relative height of the rim then we let $\varepsilon=H / L \ll 1$.

The dimensionless corotational Jeffreys model can then be written as

$$
\begin{aligned}
& \left(1+\lambda_{1} \frac{d}{d t}\right) \tau^{x x}-\lambda_{1}\left(\frac{\partial_{z} u}{\varepsilon^{2}}-\partial_{x} w\right) \tau^{x z} \\
= & 2 \mu\left(1+\lambda_{2} \frac{d}{d t}\right) \partial_{x} u-\lambda_{2} \mu\left(\frac{\left(\partial_{z} u\right)^{2}}{\varepsilon^{2}}-\varepsilon^{2}\left(\partial_{x} w\right)^{2}\right) \\
& \left(1+\lambda_{1} \frac{d}{d t}\right) \tau^{z z}+\lambda_{1}\left(\frac{\partial_{z} u}{\varepsilon^{2}}-\partial_{x} w\right) \tau^{x z} \\
= & 2 \mu\left(1+\lambda_{2} \frac{d}{d t}\right) \partial_{z} w+\lambda_{2} \mu\left(\frac{\left(\partial_{z} u\right)^{2}}{\varepsilon^{2}}-\varepsilon^{2}\left(\partial_{x} w\right)^{2}\right) \\
( & \left.+\lambda_{1} \frac{d}{d t}\right) \tau^{x z}+\frac{\lambda_{1}}{2}\left(\partial_{z} u-\varepsilon^{2} \partial_{x} w\right)\left(\tau^{x x}-\tau^{z z}\right) \\
= & \mu\left(1+\lambda_{2} \frac{d}{d t}\right)\left(\partial_{z} u+\varepsilon^{2} \partial_{x} w\right)+\lambda_{2} \mu\left(\partial_{z} u-\varepsilon^{2} \partial_{x} w\right) \partial_{x} u
\end{aligned}
$$

These equations are coupled to the nondimensional governing equations, which read in the strong slip case as

$$
\partial_{x} u+\partial_{z} w=0
$$

$$
\varepsilon^{2} \operatorname{Re}^{*} \frac{\mathrm{d} u}{\mathrm{~d} t}=-\varepsilon^{2} \partial_{x} p+\varepsilon^{2} \partial_{x} \tau^{x x}+\partial_{z} \tau^{x z}
$$

and

$$
\varepsilon^{2} \operatorname{Re}^{*} \frac{\mathrm{d} w}{\mathrm{~d} t}=-\partial_{z} p+\partial_{x} \tau^{x z}+\partial_{z} \tau^{z z}
$$

The corresponding scaled boundary conditions at $z=$ $h(x, t)$ are given by

$$
-p_{R}+\frac{\varepsilon^{2} \partial_{x} h^{2} \tau^{x x}-2 \partial_{x} h \tau^{x z}+\tau^{z z}}{1+\varepsilon^{2}\left(\partial_{x} h\right)^{2}}=\frac{\partial_{x x} h}{\left(1+\varepsilon^{2}\left(\partial_{x} h\right)^{2}\right)^{3 / 2}}
$$

and

$$
\tau^{x z}\left(1-\varepsilon^{2}\left(\partial_{x} h\right)^{2}\right)-\varepsilon^{2} \partial_{x} h \bar{\tau}=0 .
$$

In the last equation we have introduced the first normal stress difference $\bar{\tau}$ (commonly denoted by $N_{1}$ [ 6 ]

$$
\bar{\tau} \equiv \tau^{x x}-\tau^{z z}
$$

Finally, the kinematic condition reads

$$
\partial_{t} h=-\nabla \cdot \int_{0}^{h} d z \mathbf{u}
$$

Note that $p_{R}=p+V(h)$, where $V(h)$ here denotes a disjoining pressure due to dispersion forces across the film.

At the substrate, $z=0$, we have as usual the impermeability condition $w=0$ and the slip condition $u=b \tau^{x z}$. In the strong-slip regime we have $b=\beta_{s} / \varepsilon^{2}, \beta_{s}$ being the slip length parameter of order $\mathcal{O}\left(\varepsilon^{0}\right)$, see [2] for details.

For the derivation of the lubrication approximation we assume that the dynamic variables can be given in form of the asymptotic expansions

$$
\begin{aligned}
\left(u, w, h, p, \tau^{i j}\right)= & \left(u_{0}, w_{0}, h, p_{0}, \tau_{0}^{i j}\right) \\
& +\varepsilon^{2}\left(u_{1}, w_{1}, h_{1}, p_{1}, \tau_{1}^{i j}\right)+O\left(\varepsilon^{4}\right) .
\end{aligned}
$$

To leading order in $\varepsilon$ we require from equations (8) and (9) that

$$
\partial_{z} u_{0}=0 \quad \text { or } \quad \tau_{0}^{x z}=\frac{\lambda_{2}}{\lambda_{1}} \partial_{z} u_{0} .
$$

The governing equations are, to leading order,

$$
\begin{aligned}
& \partial_{x} u_{0}+\partial_{z} w_{0}=0, \\
& \partial_{z} \tau_{0}^{x z}=0, \\
& \partial_{z} p_{0}=\partial_{x} \tau_{0}^{x z}+\partial_{z} \tau_{0}^{z z},
\end{aligned}
$$

and the leading order boundary conditions at $z=h_{0}$ are

$$
\begin{aligned}
& \tau_{0}^{x z}=0 \\
& p_{R 0}-2\left(\frac{\tau_{0}^{z z}}{2}-\partial_{x} h_{0} \tau_{0}^{x z}\right)+\partial_{x x} h_{0}=0, \\
& \partial_{t} h_{0}-w_{0}+u_{0} \partial_{x} h_{0}=0 .
\end{aligned}
$$


Leading order boundary conditions at the substrate are

$$
w_{0}=0 \text { and } \tau_{0}^{x z}=0 .
$$

We now integrate (21) with respect to $z$ and use the boundary conditions (23) to find

$$
\tau_{0}^{x z}=0
$$

With the constitutive equations (19) and the boundary conditions (26) we are led to the plug flow condition $\partial_{z} u_{0}=$ 0 .

The next order equations for (8), (9) are then given by

$$
\begin{aligned}
& \left(1+\lambda_{1} \frac{d^{0}}{d t}\right) \tau_{0}^{x x}=2\left(1+\lambda_{2} \frac{d^{0}}{d t}\right) \partial_{x} u_{0}, \\
& \left(1+\lambda_{1} \frac{d^{0}}{d t}\right) \tau_{0}^{z z}=2\left(1+\lambda_{2} \frac{d^{0}}{d t}\right) \partial_{z} w_{0}
\end{aligned}
$$

where $d^{0} / d t=\partial_{t}+u_{0} \partial_{x}+w_{0} \partial_{z}$. Also, from (22), we find with the solution (27) and the boundary condition (24) for the pressure at the liquid surface $z=h_{0}$

$$
p_{0}=\tau_{0}^{z z}-\partial_{x x} h_{0}-V\left(h_{0}\right) \text {. }
$$

The next order $\left(O\left(\varepsilon^{2}\right)\right) u$-momentum equation is

$$
\operatorname{Re}^{*} \frac{d^{0} u_{0}}{d t}=-\partial_{x} p_{0}+\partial_{x} \tau_{0}^{x x}+\partial_{z} \tau_{1}^{x z}
$$

Using (30) and denoting $u_{0}=f(x, t)$ we obtain

$$
\operatorname{Re}^{*}\left(\partial_{t} f+f \partial_{x} f\right)=\partial_{x} \overline{\tau_{0}}+\partial_{x}\left(\partial_{x x} h_{0}+V\right)+\partial_{z} \tau_{1}^{x z} .
$$

Integrating this last equation from 0 to $h_{0}$ we find, using the slip boundary conditions to the next order, $\tau_{1}^{x z}=$ $f / \beta_{s}$,

$$
\begin{aligned}
h_{0} \operatorname{Re}^{*}\left(\partial_{t} f+f \partial_{x} f\right) & =\partial_{x} \int_{0}^{h_{0}} d z \overline{\tau_{0}}-\left.\overline{\tau_{0}}\right|_{z=h_{0}} \partial_{x} h_{0} \\
& +h_{0} \partial_{x}\left(\partial_{x x} h_{0}+V\right)+\left.\tau_{1}^{x z}\right|_{z=h_{0}}-\frac{f}{\beta_{s}} .
\end{aligned}
$$

The next order tangential stress boundary condition at $z=h_{0}$ is

$$
\tau_{1}^{x z}=\overline{\tau_{0}} \partial_{x} h_{0}
$$

Hence

$$
\begin{aligned}
h_{0} \operatorname{Re}^{*}\left(\partial_{t} f+f \partial_{x} f\right) & =\partial_{x} \int_{0}^{h_{0}} d z \overline{\tau_{0}} \\
& +h_{0} \partial_{x}\left(\partial_{x x} h_{0}+V\right)-\frac{f}{\beta_{s}} .
\end{aligned}
$$

From equations (28) and (29) we obtain an equation for the difference of the diagonal terms of the stress tensor,

$$
\begin{aligned}
& \left(1+\lambda_{1} \partial_{t}+\lambda_{1} f \partial_{x}-\lambda_{1} z \partial_{x} f \partial_{z}\right) \overline{\tau_{0}} \\
& =4\left(\partial_{x} f+\lambda_{2} \partial_{t} \partial_{x} f+\lambda_{2} f\left(\partial_{x} f\right)^{2}-\lambda_{2} z \partial_{x} f \partial_{z} \partial_{x} f\right)
\end{aligned}
$$

We now define a film-average $S$ of $\overline{\tau_{0}}$ as

$$
S \equiv \frac{1}{4 h_{0}} \int_{0}^{h_{0}} d z \overline{\tau_{0}} .
$$

We denote the RHS of (35) by $G(x, t)$ and observe that the last term is zero. Integrating (35) with respect to $z$ then yields

$$
\begin{aligned}
& 4 h_{0} S+\lambda_{1} \partial_{t}\left(4 h_{0} S\right)+\lambda_{1} f \partial_{x}\left(4 h_{0} S\right)+\lambda_{1}\left(4 h_{0} S\right) \partial_{x} f \\
& -\left.\lambda_{1} \overline{\tau_{0}}\right|_{z=h_{0}}\left(\partial_{t} h_{0}+f \partial_{x} h_{0}+h_{0} \partial_{x} f\right)=h_{0} G(x, t) .
\end{aligned}
$$

Using the kinematic condition to leading order

$$
\partial_{t} h_{0}+\partial_{x}\left(f h_{0}\right)=0
$$

and employing the definition of $G(x, t)$ then finally leads to

$$
\left(1+\lambda_{1} \partial_{t}+\lambda_{1} f \partial_{x}\right) S=\left(\partial_{x}+\lambda_{2} \partial_{t} \partial_{x}+\lambda_{2} f \partial_{x x}\right) f
$$

The lubrication model can finally be written as

$$
h_{0} \operatorname{Re}^{*}\left(\partial_{t} f+f \partial_{x} f\right)=\partial_{x}\left(4 h_{0} S\right)+h_{0} \partial_{x}\left(\partial_{x x} h_{0}+V\right)-\frac{f}{\beta_{s}}
$$

together with (38) and (39). Note that only the advective non-linearities, but not the corotational non-linearities appear in (39). This implies, that using the upper or lower advective derivative instead of the Jaumann derivative (leading to Oldroyd's model A or B) leads to the same one-dimensional thin film equation.

We are currently investigating the effect of viscoelastic relaxation in strongly slipping films on the morphology of dewetting rims.

\section{References}

1. R. Fetzer, K. Jacobs, A. Münch, B. Wagner and T. P. Witelski, Phys. Rev. Lett. 95, 127801 (2005)

2. A. Münch, B. A. Wagner and T. P. Witelski, J. Engr. Math. 53(3-4), 359-383, (2005)

3. K. Kargupta, A. Sharma and R. Khanna, Langmuir 20, 244 (2004)

4. M. Rauscher, A, Münch, B. Wagner and R. Blossey, Eur. Phys. J. E 17, 373 (2005)

5. R. Blossey, A. Münch, M. Rauscher, B. Wagner, submitted (2006)

6. R. B. Bird, R.C. Armstrong and O. Hassager, Dynamics of polymeric liquids, Vol. 1, John Wiley \& Sons, 1987; note that our sign convention is different, see ref.[4]. 